VI. Aus Prof. Dr. L. Landan's Franenklinik in Berlin.

\section{Zur Symptomatologie und Therapie der Retroflexio uteri.*)}

\section{Von Dr. Curt Freudenherg, Assistenten der Klinik.}

Nachdem Velde und Strassmann ${ }^{1}$ ) im Jahre 1895 zuerst über schwere Geburtsstörungen infolge voraufgegangener Antefixation des Uterns bericlitet hatten, folgte bald eine nicht allzu kleine Reihe derartiger Veröffentlichungen. Es war danach zn er warten, dass der Eifer für die operative Behandlung der Retrodeviationen des Uterus etwas erkalten würde. Bisher scheint dies jedoch nicht der Fall zu sein. Die ursprïnglich bevorzugten Operationsmethoden - Ventro- und Vaginofixation - wurden allerdings zum Theil ganz verlassen, zum Theil in ihrer Anwendung erheblich beschränkt; aber an ihre Stelle traten sofort neue Operationsmethoden, und es entspann sicl ein heftiger Streit darüber, welche nunmehr die beste sei.

Das Geränsch dieses Streites ïbertönte fast vollständig die Stimmen derer, welche in ihren Anschannngen ïber die Bedeutung der Retroflexio nteri erheblich von der gültigen Lehre abwichen. Insbesondere aber nahm der Theil der Fachpresse, welcher den allgemeinen Interessen des praktischen Arztes dient, ïberwiegend keine Notiz von den diesbezüglichen Arbeiten Theilhaber's'), Salin's") und Jenkins't). Und doch hätten diese Arbeiten eine Erwahnung schon deshalb verdient, weil bei der Diseussion über dieselben nothwendigerweise eine Frage erörtert werden musste, welche für den allgemeinen Praktiker wichtiger ist, als der Streit um die beste Operationsmethode; diese Frage lautet: welche Erfolge erzielt die nichtoperative Therapie gegenüber den Beschwerden derjenigen Franen, welche mit einer Retroflexio nteri behaftet sind?

Zur Erörterung dieser Frage, sowie einiger damit zusammenlängender Punkte möchte ich einen kleinen Beitrag geben, an der Hand der Erfahrungen, welche in der Poliklinik des Herrn Prof. Dr. L. Landan gemacht wurden. Besonders sollen dabei berücksichtigt werden die in der Zeit vom 1. Januar 1895 bis 30 . Juni 1896 nell aufgenommenen Kranken, ïber deren Befinden wir in der letzten Zeit eine Enquête veranstalteten, indem wir sie durch Karte zul einer nochmaligen Vorstellung anfforderten.

In dem genannten Zeitraum kamen insgesammt 423 Kranke zur poliklinischen Anfnahme, bei welchen eine Retrodeviation des Uterus constatirt wurde. Es sei hier gleich bemerkt, dass in den folgenden Ausführungen ein Unterschied zwischen Retroflexio und Retroversio im allgemeinen nicht gemacht wird, dass daher n̈berall, wo nicht das Gegentheil aus dem Znsammenhange hervorgeht, unter Retroflexio beide Arten der Rückwärtslagerung verstanden werden.

In jedem einzelnen Fall waren wir zunächst bemïht, ein Urtheil darüber zu gewinnen, ob die Beschwerden der Kranken wirklich durch die Lageveränderung bedingt seien oder nicht. Dazı war in erster Linie festzustellen, ob die Retroflexio den einzigen von der Norm abweichenden Befund darstelle, oder ob daneben andere pathologische Veränderungen zunächst in den Genitalien nachzuweisen seien. Wir begnügten uns aber nicht mit einer Feststellung des Genitalbefundes, sondern nahmen in der Regel - im letzten Theil der Beobachtungszeit ausnahmslos - eine Palpation der Abdominalorgane und eine Prüfung des Urins vor; die übrigen Körperorgane wurden untersucht, soweit Anzeichen fïr eine Erkrankung derselben vorhanden waren.

Wie nöthig eine solche Vorsicht ist, zeigten eklatant drei Fälle, über die ich kurz berichten will.

1. Frau B. Tr. sucht am 17. Januar 1895 die Poliklinik auf. Patientin ist in den letzten Jahren zweimal per vaginam, einmal per laparotomiam operirt worden. Die Beschwerden bestehen unverändert fort: heftige Schmerzen im Leib und Rückgrat, blitzartige Schmerzen in Beinen und Armen, Globus. Grösste Schmerzen zur Zeit der Periode. Häufig Erbrechen und "Magenkrämpfe".

Genitalbefund: Laparotomienarbe. Vaginaefixationsnarbe. Uterus ante-

*) Bei der Redaction am 10. April 1897 eingegangen.

1) Verhandlungen der Gesellschaft der Charitéärzte. Berliner klinische Wochenschrift 1896, No. 36 .

2) „Die Therapie der Retroflexio uteri.“ Monatsschrift fuir Geburtshülfe und Gynäkologie, Bd. II, sowie „Pathologie und Therapie der Retroflexio uteri.“ Ibid. Bd. III.

3) Citirt nach Josephson, Schwedische geburtshülfliche und gynäkologische Litteratur aus dem Jahre 1894. Monatsschrift für Geburtshülfe und Gynäkologie, Bd. II.

*) Retroflexion of the uterus and its treatment. Glasgow medical Journal 1896, Februar. (Glasgow south medical society.) flectirt, von normaler Grösse, mit der Bauchnarbe fest verwachsen. Beide Ovarien fuhlbar, wenig vergrössert.

Patientin drängte auf ihre Aufnahme in die Klinik, ,um sich die Gebärmutter herausnehmen zu lassen." Hier wurde reflectorische Pupillenstarre, Fehlen der Patellarreflexe festgestellt. Diagnose: T'abes dorsalis. Patientin entlassen. Es trat dann später noch ein syphilitisches Geschwïr (zerfallenes Gummi) am linken Zungeurande auf, welches vielleicht auf dio Aetiologie des Nervenleidens hinweist.

2. Fran A. Fr. sucht am 27. September 1895 die Poliklinik auf. Bei der Patientin sind in den letzten Jahren von anderer Seite drei Vorfallbezw. Scheidenoperationen gemacht worden. Seit der letzten Operation, bei welcher sie nach ihrer Meinung „zu eng vernäht worden war“, stärkere Beschwerden: Gefühl, als ob sie ersticken müsse u. s. w.

Genitalbefund: Am Damm Narben von plastischen Operationen. Vaginaefixationsnarbe, mit welcher der anteflectirte, etwas descendirte Uterus fest verwachsen ist. Sonst normale Verhältnisse.

Dass Patientin psychisch nicht normal war, schien uns von vornherein nach ihrem ganzen Verhalten, insbesondere den Klagen, klar; sie schien auch nach ihren Angaben unuittelbar nach der letzten Operation einen heftigen Erregungszustand durchgemacht zu haben. Wir stellten zunächst die Wahrscheinlichkeitsdiagnose: Paranoia. Die weitere Beobachtıng ergab jedoch, dass es sich um eine Dementia paralytica handelte es trat nach etwa sieben Monaten die charakteristische Sprachstörnng bei rapider Abnahme der Intelligenz ein.

3. Frl. L. R. wird uns am 17. September 1895 von Herrn Collegen Dr. D. Munter mit der Diagnose: chronische Paranoia zugeführt. Die Erkrankung besteht sicher bereits seit 1894; Patientin suchte damals ärztliche Hitlfe anf wegen heftiger Beschwerden im Unterleib (Geftihl des Herausfallens eines Gegenstandes u. s. w.). Als Ursache der Erkrankung sah Patientin die zll therapeutischen Zwecken vorgenommenen Manipulationen eiues hiesigen Homöopathen an, der ihr dabei „die Gebärmutter verdreht hïtte." Die Diagnose des behandelnden Arztes war schon damals Paranoia chronica, daneben bestand Retroflexio. Patientin suchte dann ohne Wissen ihres Arztes einen Gynäkologen auf, der eine Operation vornahm. Wir constatirten bei der Patientin als Genitalbefuud: Vaginaefixationsnarbe, Retroflexio uteri mobilis. Im Einvertändniss mit Herrn Collegen Munter machten wir den wenig aussichtsvollen Versuch, durch Beseitigung der Lageanomalie auf das psychische Verbalten einzuwirken. Es gelang leicht, durch ein Pessar Lagecorrectur zu erhalten. Die Psyche und die örtlichen Beschwerden blieben uubeeinflusst.

Wir halten es für fast unzweifelhaft, dass in diesen drei Fällen ans Anlass von Beschwerden, deren Ursache im Centralnervensystem lag, eine ganze Reihe gynäkologischer Operationen vorgenommen worden ist. Wie oft mag dasselbe bei hysterischen, bezw. neurasthenischen Kranken geschehen sein!

Doch kehren wir zn der allgemeinen Betrachtung der gesammten, in dem betreffenden Zeitranm beobachteten Retroflexionen zurück. Unter den 423 Kranken zeigten 84 bewegliche Retroflexioversionen ohne weitere Complication. In 339 Fällen war die Lageveränderung durch eine andere Erkrankung complicirt. Unter diesen waren 119 fixirte Retroflexionen; sonstige Adnexerkrankungen d. h. abgesehen von der Pelviperitonitis, bezw. Parametritis, welche die Fixation bedingt - wurden constatirt in 111 Fällen, Scheidenoder Gebärmnttervorfall in 63 Fällen, Venter pendulıs in $38 \mathrm{Fällen}$, einseitige Wanderniere in 53, doppelseitige in 4 Fällen, Wanderleber in 18 Fällen, einmal Wandermilz, sonstige Erkrankungen in 69 Fällen.

Naturgemäss waren in einer Reihe von Fällen, wie ja schon die Addition ergiebt, mehrere der anfgezählten Complicationen gleichzeitig vorhanden. Es ist ferner zu dieser Zusammenstellnng zu bemerken, dass die Zahl der Fälle, welche mit Wanderniere oder Wanderleber, also Theilerscheinungen allgemeiner Enteroptose, complicirt sind, deswegen zu niedrig veranschlagt ist, weil, wie erwahnt, nur in dem letzten Theile der Beobachtungszeit ansnahmslos jede Kranke einer diesbezüglichen Untersuchung unterworfen wurde. Während im Jahre 1895 in unseren Journalen unter 258 Kranken mit Retrodeviationen des Uterus nur bei 14 Ren mobilis simplex, bei einer Ren mobilis duplex, bei sieben Wanderleber notirt wurde, sind die entsprechenden Zahlen für das erste Halbjahr 1896, in welchem diese Untersuchung, wenigstens nach der Sitzung der Berliner medicinischen Gesellschaft vom 25. März, regelmässig vorgenommen wurde: unter 165 Fällen von Retroflexio $39 \mathrm{Mal}$ einseitige Wanderniere, $3 \mathrm{Mal}$ doppelseitige, 11 Mal Wanderleber. Selbst diese Zahlen sind noch zu niedrig; die Nachuntersuchnng von 162 Kranken, die mit Retroflexio uteri in Behandlung gestanden hatten, ergab $66 \mathrm{Mal}$ einseitige Wanderniere, $15 \mathrm{Mal}$ doppelseitige, $18 \mathrm{Mal}$ Wanderleber.

Es sei dies ausdrücklich erwähnt, weil in der Sitzung der Berliner medicinischen Gesellschaft vom 25. März 1896 Mackenrodt5) behauptete, dass nur ungefähr jeder zehnte Fall von Retro-

5) Berl. kliu. Woehenschr. 1896, No. $1 \%$. 
flexio sich als Theilerscheinung der Enteroptose auffassen lässt; die vorstehend genannten Zahlen geben denn doch ein etwas anderes Bild.

Aus dem höheren Antheil der Wandernieren u. s. w. ergiebt sicl, dass umgekehrt die oben gegebene Zahl der "reinen“ Retroflexionen als eine $\mathrm{zu}$ hohe zu betrachten ist. Die regelmässig vorgenommene Untersuchung hätte zweifellos auch in einem Theil dieser Fälle Complication mit Wanderniere oder Wanderleber ergeben; bei den 162 nachuntersuchten Kranken fanden sich, selbst wenn inan die unbedeutenderen Affectionen übersah, nur 23 Lageveränderungen ohne Complication. Es sei zugestanden, dass durch Unterlassung der regelmässigen Untersuchung auf Wanderniere u. s. w. ein Fehler begangen worden ist, welcher sicher auch in einer Reihe von Fällen den Erfolg der Therapie ungünstig beeinflusst hat; es ist auch seitdem in der Poliklinik zur ausnahmslos befolgten Regel geworden, jede Kranke sofort bei ihrer Aufnahme auf Wanderniere u. s. w. zu untersuchen.

Zu der vorstehenden Statistik ist ferner zu bemerken, dass wir unter der Rubrik "Complicationen mit sonstigen Erkrankungen“ nicht etwa alle die Fälle zusammengefasst haben, in welchen ein leichterer oder schwererer Grad von Anämie zu constatiren war, oder welche uns der Hysterie, bezw. Neurasthenie verdächtig erschienen. Unter den 70 Fällen dieser Rubrik befinden sich nur drei Hysterische, welche entweder in der Poliklinik typische hysterische Anfälle bekommen hatten, oder uns von Nervenärzten schon mit dieser Diagnose zugesandt waren. Bei allen anderen handelte es sich um Erkrankungen mit deutlichem Befund. Trotzdem wir bei einer Reihe von Kranken nicht in der Lageveränderung der Gebärmutter, sondern in der allgemeinen Anämie, bezw. Neurasthenie die Ursache der Beschwerden vermutheten, und trotzdem uns in einer grossen Zahl dieser Fälle der Erfolg recht gab, nahmen wir doch davon Abstand, diese Fälle besonders zu rubriciren. Wir wollten auch den leisesten Verdacht vermeiden, als ob wir, einer Theorie zu Liebe, den Thatsachen Zwang anthun und alle diejenigen Kranken als nervös, bezw. hysterisch qualificiren wollten, bei welchen wir ausser der Retroflexio einen pathologischen Befund nicht erheben konnten.

Bei der grossen Mehrzahl der Fälle, in welchen die Retroflexio durch eine andere Erkrankung complicirt war, liess sich durchaus nicht leicht entscheiden, welchen Antheil an den Beschwerden der Kranken die Lageveränderung, und welchen die Complication hatte: denn es kann nach den Beobachtungen, dio in der Landau'schen Klinik gemacht wurden, nicht zugegeben werden, dass die Retroversio-flexio ein wohlcharakterisirtes, in seinen Erscheinungen abgerundetes Krankheitsbild "6) darbietet. Schon Theilhaber ${ }^{7}$ ) hat ja darauf hingewiesen, dass E. Martin in seinem Werke über ,die Neigungen und Beugungen der Gebärmutter" ein Symptomenbild als charakteristisch für die Anteflexio uteri puerperalis schildert, das genaul dem hente als typisch für die Retroflexio geltenden entspricht: "Die Mehrzahl der Kranken mit Anteflexio uteri puerperalis klagt über Schmerzen im Hypochondrium, Kreuzschmerz, Drängen. Harnbeschwerden sind häufig, . . . Stuhlverstopfung findet gewöhnlich statt. Fast in allen Fällen sind heftige Blutungen vorhanden." Ganz ähnlich diesem ist auch der

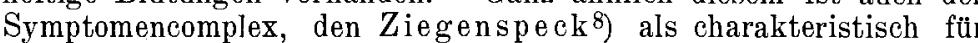
die häufige Combination von chronischer Endometritis und Parametritis schildert. Der Symptomencomplex der Retroflexio entspricht ferner daurchaus dem "syndrôme utérin“ Pozzi's, 9) d. h. der Summe von Erscheinungen, welche nach diesem bei allen Gebärmuttererkrankungen auftreten können; Pozzi selbst lässt keinen Zweifel daran auflkommen, dass dies auch seine Meinung sei, indem er die Symptomatologie der Retroflexio mit den Worten einleitet: „Syndrôme utérin, phénomènes nerveux réflexes très accentués, stérilité; tel est, en résumé, le bilan des symptômes rationnels." 10 )

Im übrigen wird wohl jeder Arzt, der ein grösseres gynäkologisches Material beobachtet, darunter auch Kranke gefunden haben, welche den sogenannten "typischen Symptomencomplex" der Retroflexio darboten und trotzdem einen anteflectirten Uterus hatten. Wenigstens boten uns derartige Fälle wiederholt Gelegenheit, zu zeigen, dass gerade bezüglich der Retroflexio uteri die Anamnese nicht zu so weitgehenden Schlussfolgerungen berechtigt, als häufig von den unsere Klinik besuchenden Studirenden und Aerzten gezogen wurden.

$\left.{ }^{6}\right)$ cf. Kustner, Normale und pathologische Lagen und Bewegungen des Uterus. Stuttgart 1885, S. 43.

7) I. c.

8) Anleitung zur Massagebehandlung (Thure-Brandt) bei Franenleiden. Berlin 1895 , S. 65.

9) ef. Traité de gynécologie. Paris 1890, S. 177.

$\left.{ }^{10}\right)$ 1. c. S. 469
Wenn aber der ganze Symptomencomplex nicht als charakteristisch für die Retroflexio angesehen werden kann, so gilt dies noch weniger von den einzelnen Symptomen. Sämmtliche $\mathrm{Be}$ schwerden, über welche Retroflexionskranke klagen, kommen einzeln, oder zu mehreren vereint, auch bei anderen Affectionen vor. Trotzdem, oder gerade deshalb, verdienen die einzelnen Symptome eine Besprechung.

Beginnen wir bei den Kreuzschmerzen, da hierüber am häufigsten von unsern Kranken Klage geführt wurde. Dass gerade die Retroflexio in hervorragendem Maasse solche Schmerzen erzeugte, war in keiner Weise ersichtlich. Im Gegentheil, soweit nicht Gravidität, Blutungen, Harnbeschwerden oder weisser Fluss die alleinigen Klagen darstellten, welche die betreffenden Frauen zur Poliklinik führten, - soweit also überhaupt Schmerzen vorhanden waren, - gaben fast alle Kranken entweder spontan oder auf Befragen Kreuzschmerzen an, ohne dass die Lage des Uterus dabei einen Unterschied machte. Immerhin schien ein Punkt nicht ganz bedeutungslos zu sein: die Lokalisation dieser Kreuzschmerzen. Nach der medicinischen Terminologie sollte man unter diesem Namen Schmerzen in der Gegend oder Höhe des Kreuzbeins verstehen. Das Volk versteht aber darunter etwas ganz anderes. Wird von einem Schlag oder Stoss „in das Kreuz" gesprochen, so ist als betroffen nicht die hintere Beckenwand anzusehen, sondern der Theil des Rückens, welcher den oberen Lendenwirbeln, bezw. den Brustwirbeln entspricht, also etwa die Taillengegend. Genau so werden Frauen, welche genau den Sitz ihrer "Kreuzschmerzen" bezeichnen sollen, nur in seltenen Fällen auf die hintere Beckellwand hinweisen; meist wird entweder die Gegend der Lendenwirbel oder sogar die hintere Thoraxwand, insbesondere die Partie unterhalb der Schulterblätter und zwischen ihnen, bezeichnet werden.

Trotzdem eine ausreichende Erklärung dafür fehlt, kann es wohl durch vielfache Beobachtungen bei den verschiedensten gynäkologischen Affectionen - insbesondere bei Beckenabscessen als sicher erwiesen angesehen werden, dass als Begleiterscheinung dieser Leiden häufig Sclimerzen im Rücken in der Höhe der Lendenwirbelsäule auftreten. Andererseits finden sich Schmerzen in dieser Gegend auch oft im Gefolge von Nephritis, Pyelitis u. s. w. Es sei dies erwähnt, weil in einer kleinen Zahl von Fällen, welche trotz heftiger "Kreuzschmerzen" einen nur mbedeutenden Genitalbefund darboten, durch die Urinuntersuchung die Erklärung dafür gegeben wurde. Auch die chronische Obstipation spielt hierbei eime Rolle. Unter allen Umständen aber erscheint es uns höchst unwahrscheinlich, dass Genitalaffectionen - von grossen Tumoren abgesehen - Schmerzen im Bereich der hinteren Thoraxwand auslösel können, insbesondere in den höheren Partien zwischen den Schulterblättern. Dagegen finden sich derartige Schmerzen als häufige Begleiterscheinungen von Hängebauch, Wanderniere, Wanderleber ${ }^{11}$ u. s. w. Wie dies zu erklären sei, bleibe dahingestellt; die Thatsache aber wird auch von andern Beobachtern bestätigt. ${ }^{12}$ ) Konnten wir in entsprechenden Fällen eine der genannten Theilerscheinungen der Enteroptose constatiren, so war auch meist die eingeleitete Therapie - Anlegung einer gut passenden Leibbinde - von Erfolg begleitet, insofern die Schmerzen gehoben oder mindestens gelindert wurden. In anderen Fällen, in welchen die Untersuchung auf Wanderniere u. s. w. negativ ausfiel, wies der Erfolg hydrotherapeutischer Maassnahmen - heisser Bäder u. s. w. - oder lokale Massage dahin, dass es sich um eine rheumatische Affection handele; auch Salin ${ }^{13}$ ) führt ja in vielen Fällen die Rückenschmerzen au eine "Myositis" der Rückenmuskeln zurück. Endlich war nicht selten Anämie als Ursache der Rückenschmerzen anzusprechen auch anämische Männer klagen ja häufig über dasselbe, und be stillenden Frauen sind meist Rückenschmerzen das erste Zeichen dafür, dass die Entwöbnung des Kindes mit Rücksicht auf die Gesundheit der Mutter nothwendig ist.

Ein grosser Theil unserer Kranken klagte ferner, sowohl bei normaler Lage des Uterus als bei Deflexionen, über Leibschmer zen. Auch hierbei erschien es angezeigt, zunächst durch Fragen genau den Sitz des Sehmerzes zu ermitteln. Bei allen Beschwerden im Bereich des Abdomen sind Frauen sehr geneigt, den Gynäkologen zu Rathe zu ziehen, in weIchem sie überhaupt vielfach nicht den Arzt für Frauenkrankheiten, sondern für kranke Frauen sehen. Wenn man in jedem Falle den Sitz der Schmerzen zu ermitteln sucht, wird man nicht allzu selten auf das Epigastrium oder die Gegend der Gallenblase hirgewiesen werden. Selbst stenocardische Erscheinungen werden von den Kranken bisweileu als „Leibschmerzen“ angegeben. Daneben besteht eine grosse Zahl von Fällen, in welchell

11) L. Landau. Die Wanderleber und der Hängebauch der Frauen. Berlin 1885 , S. 78 .

12) z. B. fur die Wanderniere von Ziegenspeck, I. c. S. 165, Strü mpell, Specielle Pathologie und Therapie. Bd. II., 2. Th., S. 92.

13) I. c. 
der Schmerz bestimmt in der Unterbauchgegend Iokalisirt wird. Für die Erklärung dieser kommen Blasen-, Mastdarm- oder Genitalaffectionen in Betracht. Bei unseren Retroflexionskranken bestanden diese Schmerzen häufig in dumpfem Druck; vielfach verbunden mit Drängen nach unten oder Gefühl, „alš ob etwas vorgefallen sei", wie dies ja auch von anderer Seite angegeben wird; mit wenigen Ausnahmen war dabei der Uterus nicht bloss retrovertirt oder retroflectirt, sondern auch descendirt, und vielfach bestand gleichzeitig Scheidenvorfall. - Es wurden aber auch häufig direkt "Schmerzen" angegeben; bei diesen Kranken fanden sich in der Regel frischere oder ältere Entzlindungsprocesse der Gebärmutter oder der Anhänge, doch kann auch unter Umständen, auf welche später einzugehen sein wird, die uncomplicirte Retroflexio mobilis solche Schmerzen hervorrufen.

Die BI utungen - Menorrhagieen oder Metrorrhagieen - und der Ausfluss, we]che häufig die Retroversio-flexio begleiten, sind Symptome einer gleichzeitig bestehenden Endometritis, welche übrigens wohl häufig auch einen Antheil an den Schmerzen hat. Diese Endometritis ist nach der herrschenden Anschaulung nicht als ein Accidens aufzufassen, sondern als direkte Folge der Lageveränderung, , bedingt durch Gefässstrangulation in den verdrehten Ligamenten " 14 ); an die Stauung schliessen sich Entzündungsprocesse an, indem "Mikroben, die unter normalen Verhältnissen im Uterus nur schwer aufkommen können, in dem kranken leicht gedeihen". - Diese Auffassung ist aber nicht einwandsfrei. Der Verlauf der Uterusgefässe macht es durchaus unwahrscheinlich, dass eine solche "Gefässstrangulation" wirklich bei Retroflexio uteri die Regel bildet. Die Blutzufuhr zum Uterus geschieht, wie Williams ${ }^{15}$ ) dargethan hat, durch zahlreiche Aeste der Uterina und Spermatica, welche - von diesen längs des Uterus, im Ligamentum latum verlaufenden Gefässen abgehend - den Uterus in einer zu seiner Längsachse im wesentlichen senkrechten Richtung umkreisen. Ganz ähnlich ist der Verlauf der Venen; bei diesen kommt aber noch hinzu, dass die Venenplexus des Uterus zahIreiche Anastomosen u. a. mit denen des Vaginalplexus haben. Die Unterbindung der Vena spermatica kann daher bei Operationen ohne Schaden ausgefïhrt werden, trotzdem normal der Abfluss des Blutes aus dem Uterus hauptsächlich durch die Spermatica geschieht (the return is effected usually and chiefly through the ovarian veins). Williams selbst erkennt die Möglichkeit einer Abflussstörung nur für den Fall an, dass der Fundus sich im Cavum Douglasii befindet; in Fällen dieser Art, die er als Hernien, nicht als Retroflexionen bezeichnen möchte, kann durch die Ligamenta sacro-uterina eine Constriction am Halse des "Bruchsackes" ausgeübt werden. Das sind aber extreme Ausnahmefälle, die Folgerungen auf die übergrosse Zahl der gewöhnlichen Retroflexionen nicht gestatten. Für diese letzteren gilt vielmehr der Ausspruch, mit dem Williams schliesst: ,the acutest flexion does not interfere with the flow of blood from the uterus".

Diese anatomischen Darlegungen bedürfen eigentlich keines Zusatzes. Nur auf einen Punkt könnte vielleicht noch hingewiesen werden. Wenn selbst die Unterbindung der Vena spermatica, also die plötzliche Unterbrechung des hauptsächlich abführenden Gefässes, keine Störung zu erzeugen vermag, so kann eine solche Wirkung wohl kaum einer Lageveränderung zugeschrieben werden, die sicher in den allermeisten Fällen sich Iangsam herausbildet, sei es im Wochenbett, sei es in der Entwickelungszeit. Gerade bei Circulationsverhältnissen, die an sich sehon so vollkommen sind, besteht hier unzweifelhaft die Möglichkeit eines Ausgleichs auf dem Wege des Collateralkreislaufes.

Theoretisch steht also die Lehre, dass die Retroflexio Blutstauung und damit günstige Bedingungen für weitere Erkrankungen schaffe, bisher auf recht schwachen Füssen. Auch sonst ist ein stringenter Beweis nicht einmal versucht. Ein solcher wäre es z. B. wenn dargethan werden könnte, dass eitrige Adnexerkrankungen relativ häufig mit Retroflexio uteri vergesellschaftet sind. Die Erfahrungen unserer Klinik lassen jedoch einen Versuch in dieser Richtung wenig. aussichtsvoll erscheinen.

Im übrigen sind verstärkte Blutungen zwar eine häufige, indess keineswegs eine regelmässige Begleiterscheinung der Retroflexio uteri, wie auch schon Theilhaber nachgewiesen hat. Von 129 Kranken, bei welchen wir bei der Nachuntersuchung Fortbestand der Retroflexio constatirten, bezeichneten ihre Menstruation als stark 37 , als mässig 55 , als schwach $17 ; 17$ waren klimakterisch, 3 anderweitig amenorrhoisch.

Dem entsprach es, dass unter 174 Kranken, die sich auf

14) Mackenrodt, Die Vesicofixatio uteri. Berliner klinische Wochenschrift 1896, No. 49.

15) On the circulation in the uterus, with some of its anatomical and pathological bearings. Transactions of the Obstretical Society of London. XXVII, 1886. unsere Aufforderung vorstellten, von der Aufnahme an bis zur Nachuntersuchung nur bei 14 eine Ausräumung bezw. Ausschabung der Gebärmutter vorgenommen wurde; in diese Zahl sind 6 Aborte mit einbegriffen.

Jedenfalls glauben wir danach sagen $\mathrm{zu}$ könffen, dass bei unseren Kranken die Endometritis corporis keine so häufige $\mathrm{Be}-$ gleiterscheinung der Retroflexio war, als bei den operativ behandelten Kranken Dührssen's ${ }^{16}$ ), der bei $99 \%$ Endometritis feststellte. Wenn im übrigen Dührssen aus der Nothwendigkeit, ein Curettement vorzunehmen, auch die Berechtigung herleitet, eine Vaginofixatio anzuschliessen, weil nach seiner Statistik „die Ausschabung und Vaginofixatio nicht gefährlicher ist, als die Ausschabung allein", so kann heute diese Deduction nicht mehr als richtig gelten, nachdem die Thatsachen die Ietzte Prämisse als falsch nachgewiesen haben.

(Fortsetzung folgt.) 